\title{
Theoretical Analysis of Poly(difluoroacetylene)
}

\author{
Ulrike Salzner* \\ Department of Chemistry, Bilkent University, 06533 Bilkent, Ankara, Turkey
}

Received: September 27, 2002; In Final Form: December 3, 2002

\begin{abstract}
The electronic structure of poly(difluoroacetylene) was investigated with the aim of determining whether it is a good candidate for a conducting polymer with high n-type conductivity. Positions of valence and conduction bands and bandwidths indicate that planar all-trans poly(difluoroacetylene) is p- and n-dopable and that onchain mobility of electrons and holes is high. Various geometries of oligomers with eight carbon atoms were optimized and compared to those of decacyanooctatetraene and octatetraene. Decafluorooctatetraene has a tendency to adopt nonplanar structures, but the planar trans form lies only $5.44 \mathrm{kcal} / \mathrm{mol}$ above the helical minimum. Since the energy for planarization is small, poly(difluoroacetylene) might be planar in the solid state. This is in contrast to the cyano analogue for which the planar trans structure lies $23.26 \mathrm{kcal} / \mathrm{mol}$ above the helical minimum. Alternating acetylene and difluoroacetylene units give rise to planar polymers with reduced band gap. Bandwidths, ionization potential, and electron affinity are average between those of the homopolymers.
\end{abstract}

\section{Introduction}

Polyacetylene (PA) was the first conducting organic polymer, ${ }^{1}$ and PA still holds the record in conductivity among conjugated organic polymers $\left(10^{5} \mathrm{~S} / \mathrm{cm}\right.$ for stretch aligned p-doped PA). ${ }^{2}$ Technical applications have not been found for PA since it is insoluble in all solvents tested, infusible, and unstable under ambient conditions. Attempts to improve stability were unsuccessful. The reason for the instability of PA and of many other small band gap conducting organic polymers is the high energy of the electrons in the valence band, which renders the polymers to become easily oxidized. ${ }^{3}$

Introducing fluorine substituents into polymers has pronounced effects. Poly(tetrafluoroethylene) (Teflon) has high thermal stability, low surface energy and a high melting point. The stability of Teflon is due to the high strengths of the $\mathrm{C}-\mathrm{F}$ bonds and the shielding of the polymer backbone by the fluorine atoms, which prevents chemical attack. With the aim of stabilizing PA in a similar way, the effect of substituting half of the hydrogen atoms or all of hydrogen atoms by fluorine has been investigated theoretically using density functional theory (DFT). Experimentally perfluorinated oligophenylenes ${ }^{4}$ and sexithiophene ${ }^{5}$ as well as poly(difluoropyrrole) $)^{6}$ have been investigated and their properties support the above considerations. Fluorinated PA has never been made but was studied with semiempirical methods ${ }^{7}$ and at the Hartree-Fock level of theory. ${ }^{8}$ Increase in electron affinity and band gap reduction have been predicted. However, the structures were not fully optimized and it was not established whether poly(fluoroacetylene) (PFA) and poly(difluoroacetylene) (PDFA) form planar polymers. In fact, due to electrostaic repulsion between the fluorine atoms, it is likely that they do not form planar polymers.

A similar approach of modifying PA has been to attempt to use cyano groups. Like for PDFA, promising properties were predicted theoretically for planar all-trans poly(dicyanoacetylene) $(\mathrm{PDCA})^{9}($ Scheme 1). Experimental results showed,

* Corresponding author: Tel.: (312) 290-2122, Fax. (312) 266-4579. E-mail: salzner@fen.bilkent.edu.tr.

\section{SCHEME 1}<smiles>CC(C)=C(C#N)C(C#N)=C(C#N)C(C)C</smiles>

PCDA<smiles>N#CC(C#N)=C(C#N)C(C#N)=C(C#N)C(C#N)=C(C#N)C(C#N)=C(C#N)C#N</smiles>

DCOT<smiles>CC(F)=C(F)C(F)=C(C)C(C)(C)C</smiles>

PDFA<smiles>FC(F)=C(F)C(F)=C(F)C(F)=C(F)C(F)=C(F)F</smiles>

DFOT<smiles>CC(F)=CC=CC(C)C</smiles>

PFHA<smiles>C=C(F)C=CC=C(F)F</smiles>

TFOT

however, that PDCA behaves like undoped cis-PA, with immobile defects and low conductivity. The tetramer of dicyanoacetylene, decacyanooctatetraene (DCOT) (Scheme 1), is unstable in the neutral form because of its huge electron affinity. Dianions of DCOT could be crystallized as tetraphenylphosphonium salt. DCOT dianions prefer all-cis conformation and form helices in the crystal. ${ }^{10}$ To establish whether similar problems have to be expected with PDFA (Scheme 1), various structures of decafluorooctatetraene (DFOT) were optimized. The results are compared with those for octatetraene (OT) and DCOT conformers. Finally, co-oligomers of acetylene and difluoroacetylene, tetrafluorooctatetraene (TFOT), were investigated. Polymer properties of planar all-trans PDFA and alltrans copolymers of acetylene and difluoroacetylene (PFHA) were investigated by extrapolation of oligomer data. Note that PFHA differs from PFA investigated earlier. ${ }^{7,8}$ We placed $\mathrm{H}$ and $\mathrm{F}$ atoms alternating on both sides of the chain, while Yamabe et al. and Bakhshi et al. placed all fluorine atoms on the same side of the chain.

\section{Methods}

DFOT (Figure 1), DCOT (Figure 2), and octatetraene (OT) (Figure 3) were optimized starting with the four geometries 


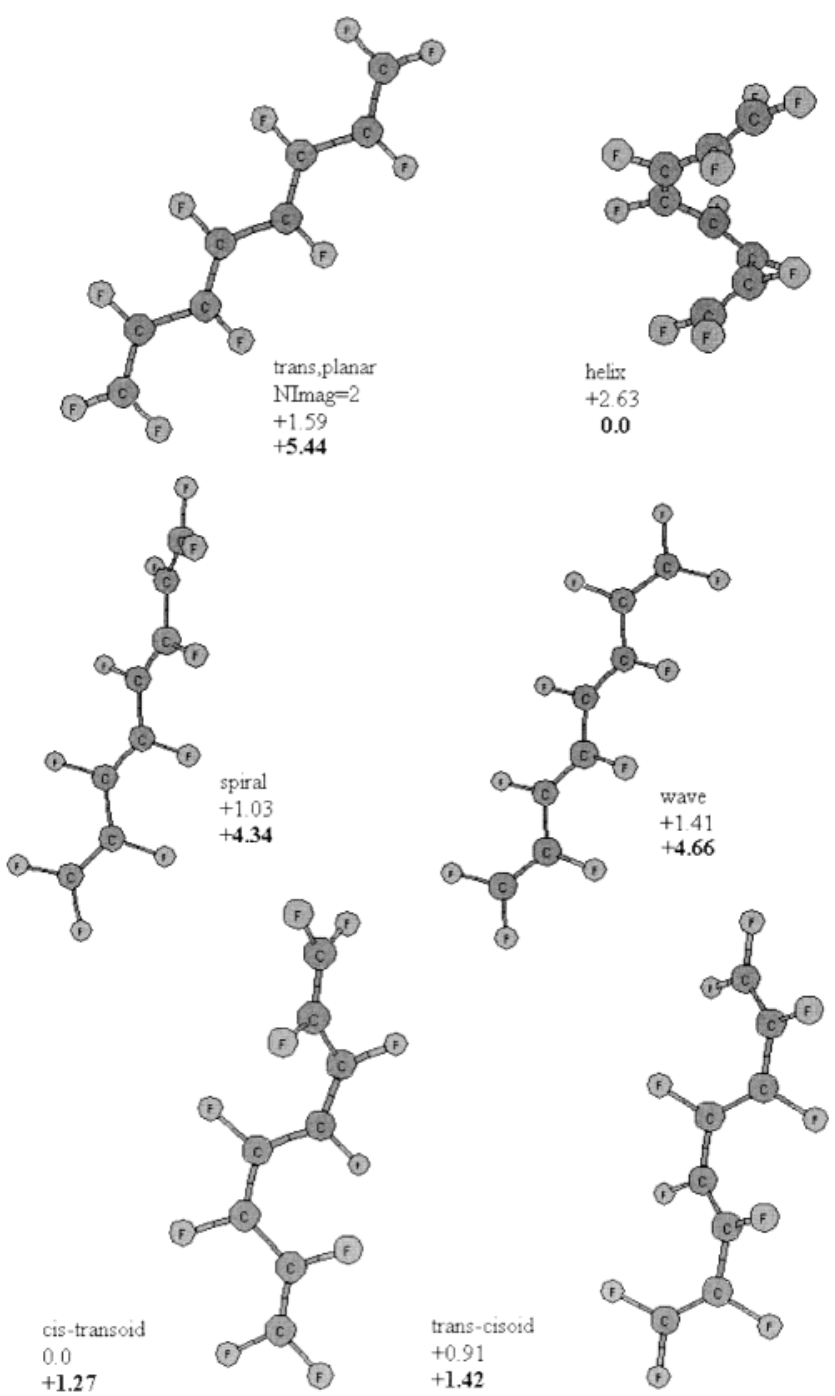

Figure 1. Optimized structures for oligomers of octatetraene (OT). Relative energies are given in $\mathrm{kcal} / \mathrm{mol}$ at the $\mathrm{B} 3 \mathrm{P} 86-30 \%$ level and at MP2 in bold face.

(trans, cis-transoid, trans-cisoid, and cis) discussed by Shirakawa. ${ }^{3}$ For TFOT (Figure 4) trans and cis structures were optimized. As in our previous investigations, we used density functional theory (DFT) with Becke's three-parameter hybrid functional, ${ }^{11}$ and Perdew and Wang's correlation functional. ${ }^{12}$ Stevens Basch Krauss pseudopotentials ${ }^{13}$ and split valence plus polarization basis sets were employed. ${ }^{13}$ For all calculations, the weight of the Hartree-Fock exchange in the DFT functional was increased to $30 \%$, since this functional yields HOMOLUMO gaps in close agreement with $\lambda_{\max }$ values from UV spectroscopy. ${ }^{14}$ The abbreviation B3P86-30\% is used for this functional. Negative orbital energies do not give accurate ionization potentials (IPs) or electron affinities (EAs) at this level of theory. The deviation is around $1 \mathrm{eV}$. Since the error is very systematic, orbital energies can be used to investigate trends reliably. Frequency calculations were carried out to verify that structures are minima on the potential energy surface. Selected structures were reoptimized at the MP2 level of theory, using the same pseudopotentials and basis sets.

Monomers through dodecamers of acetylene, difluoroacetylene, and co-oligomers of both were optimized in planar alltrans geometry. Polymer properties were evaluated by plotting data for oligomers with increasing chain length against $1 / n$, the number of repeat units, and second-degree polynomial fitting. Electronic structures were analyzed with the natural bond orbital
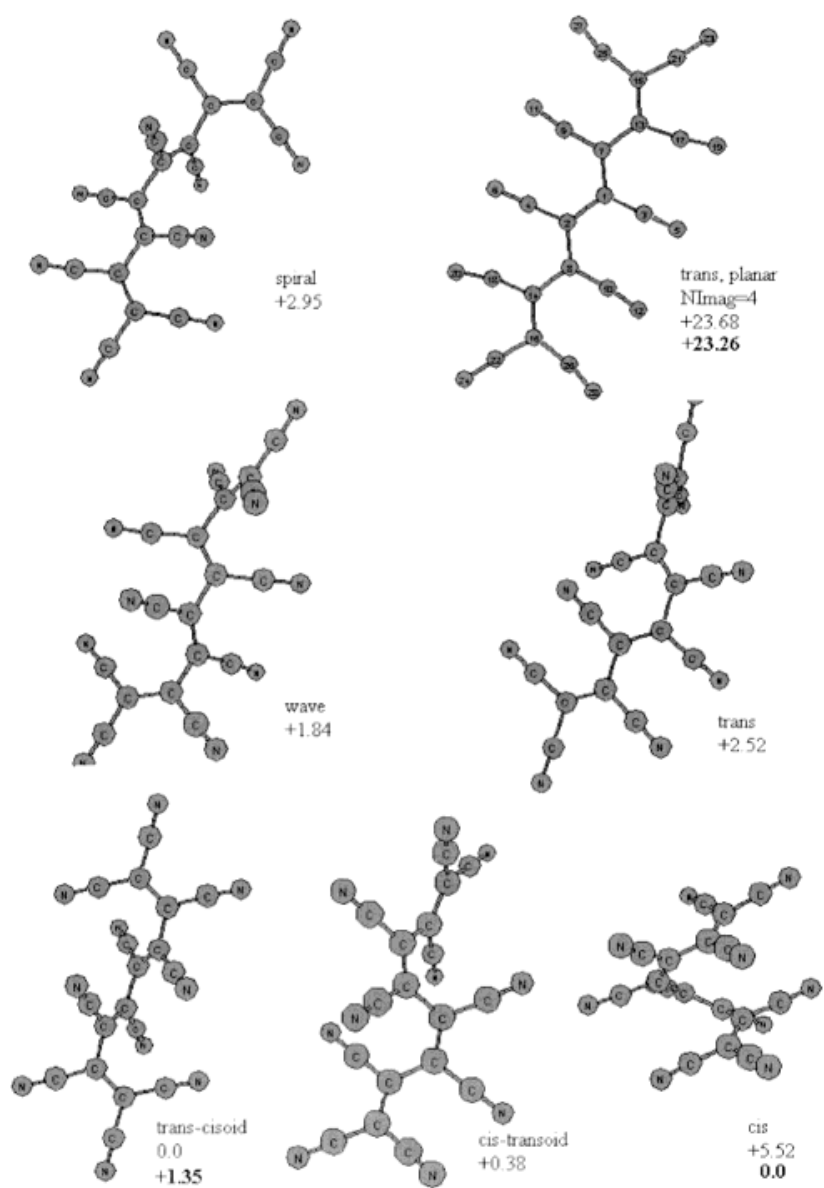

Figure 2. Optimized structures for oligomers of decacyanooctatetraene (DCOT). Relative energies are given in $\mathrm{kcal} / \mathrm{mol}$ at the $\mathrm{B} 3 \mathrm{P} 86-30 \%$ level and at MP2 in bold face.

(NBO) method. ${ }^{15-19}$ All calculations were performed with Gaussian 98 Windows and UNIX versions. ${ }^{20}$

\section{Results}

Influence of Fluoro Substitution on the Properties of Polyacetylene. Tables 1 and 2 list energy levels that extrapolate to valence and conduction band edges for acetylene and tetrafluoroacetylene oligomers. All energy levels of $\pi$-orbitals forming valence and conduction bands are plotted in Figures 5 and 6. HOMO and LUMO of tetrafluoroethylene are close to those of ethylene. The HOMO-LUMO gap is $8.64 \mathrm{eV}, 0.45$ $\mathrm{eV}$ larger than that of ethylene. The extrapolated band gaps of the polymers are almost identical, 1.62 and $1.61 \mathrm{eV}$, respectively. The conduction band edge of poly(difluoroacetylene) (PDFA) lies $1.51 \mathrm{eV}$ lower than that of PA. Therefore, PDFA is predicted to have a substantially larger EA than PA. Comparison of Figures 5 and 6 shows that this is due to a much stronger decrease of the LUMO energy upon polymerization in PDFA compared to PA. The IP of PDFA is $1.52 \mathrm{eV}$ larger than that of PA. Here the change in energy upon polymerization is stronger for PA than for PDFA. As a result, the valence bandwidth of PDFA is $1.66 \mathrm{eV}$ less than that of PA, the conduction bandwidth is $2.48 \mathrm{eV}$ larger. These data indicate that planar PDFA is a good candidate for an n-dopable material with high on-chain electron mobility.

Energy levels for alternating acetylene and difluoroacetylene units are given in Table 3. Valence and conduction bandwidths are intermediate between those of PDFA and PA. The band gap, $1.23 \mathrm{eV}$, is $0.38 \mathrm{eV}$ smaller than those of PA and of PDFA. 

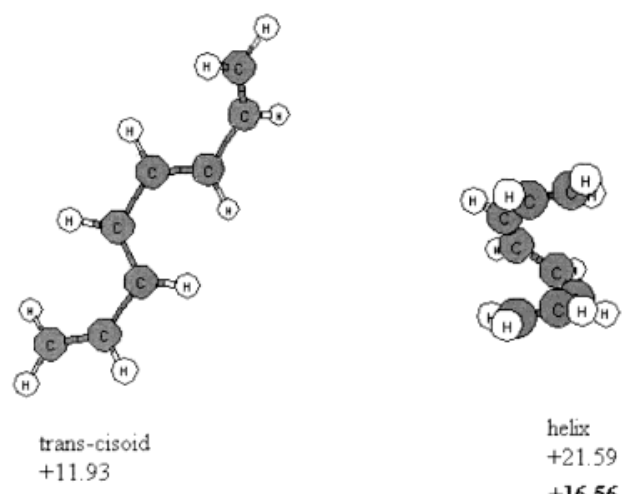

helix

$+21.59$

$+16.56$
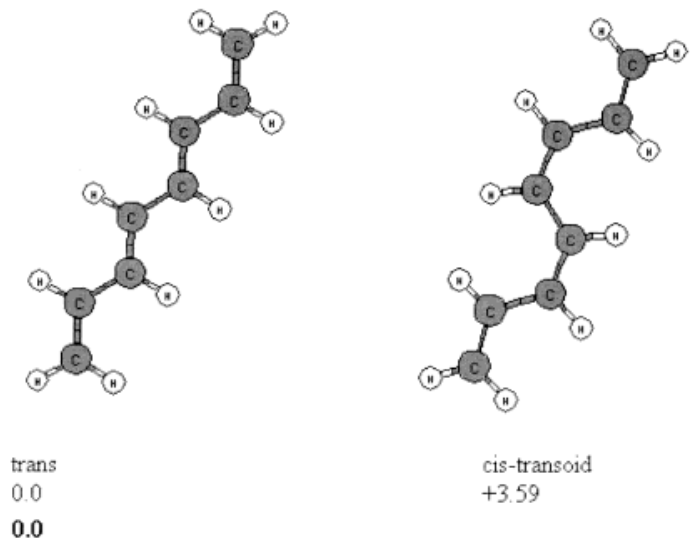

cis-transoid

$+3.59$

Figure 3. Optimized structures for oligomers of decafluorooctatetraene (DFOT). Relative energies are given in $\mathrm{kcal} / \mathrm{mol}$ at the $\mathrm{B} 3 \mathrm{P} 86-30 \%$ level and at MP2 in bold face.

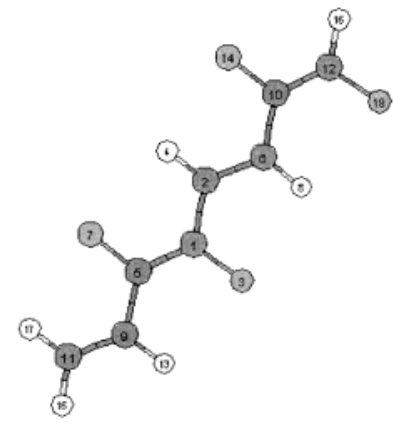

trans
0.0

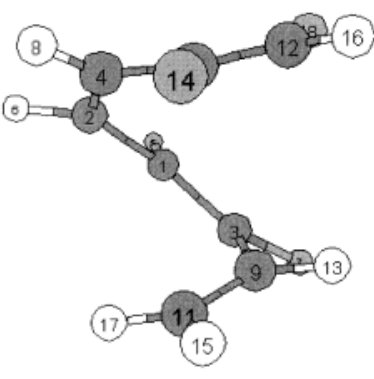

cis
+15.51
Figure 4. Optimized structures for oligomers of tetrafluorooctatetraene (TFOT). Relative energies are given in $\mathrm{kcal} / \mathrm{mol}$ at the MP2 level.

Since the theoretical band gap corresponds to $\lambda_{\max }$ rather than to the onset of absorption that is usually taken as the experimental band gap, the experimental band gap of PFHA might be around $1 \mathrm{eV}$.

Geometries of Octatetraenes. Since planar or near planar geometry is required for high on-chain mobility of charge carriers, we investigate structural preferences of decafluorooctatetraene (DFOT) and compare the relative energies to those of octatetraene (OT) and decacyanooctatetraene (DCOT). In Figure 1, geometries of DFOT are shown and relative energies are given at the B3P86-30\% and MP2 (bold face) levels of theory. Planar structures do not exist for DFOT. The all-trans analogue of OT has two imaginary frequencies and lies 1.59 $\mathrm{kcal} / \mathrm{mol}$ above the minimum at the B3P86-30\% level of

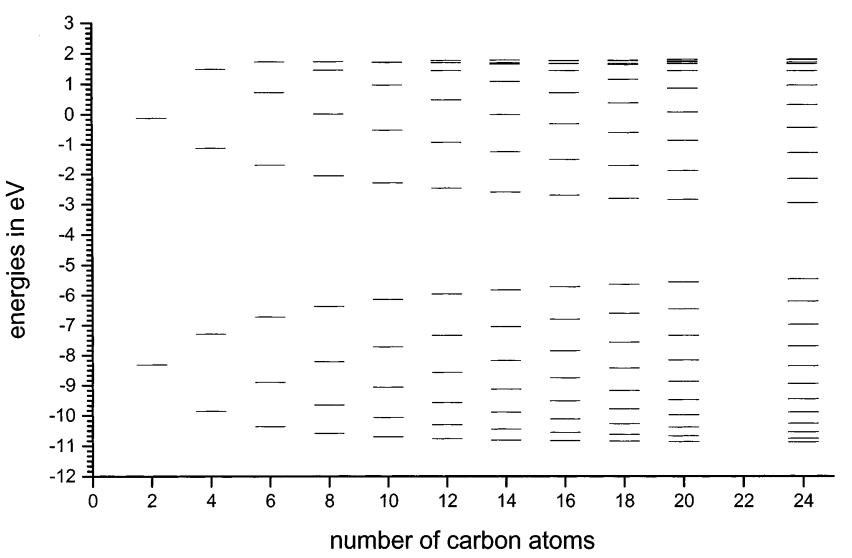

Figure 5. $\pi$-Energy levels of acetylene oligomers with 2-24 carbon atoms.

TABLE 1: Energies of Oligomer Orbitals Extrapolating toward Valence and Conduction Band Edges of PA in eV

\begin{tabular}{|c|c|c|c|c|}
\hline \# of carbons & $\begin{array}{c}\text { valence } \\
\text { band edge }\end{array}$ & HOMO & LUMO & $\begin{array}{l}\text { conduction } \\
\text { band edge }\end{array}$ \\
\hline 2 & & -8.32 & -0.13 & \\
\hline 4 & -9.86 & -7.29 & -1.13 & 1.47 \\
\hline 6 & -10.37 & -6.73 & -1.70 & 1.72 \\
\hline 8 & -10.59 & -6.38 & -2.06 & 1.72 \\
\hline 10 & -10.71 & -6.15 & -2.30 & 1.70 \\
\hline 12 & -10.77 & -5.97 & -2.48 & 1.75 \\
\hline 14 & -10.82 & -5.84 & -2.61 & 1.75 \\
\hline 16 & -10.84 & -5.74 & -2.72 & 1.74 \\
\hline 18 & -10.86 & -5.66 & -2.82 & 1.74 \\
\hline 20 & -10.88 & -5.59 & -2.87 & 1.77 \\
\hline 24 & -10.89 & -5.49 & -2.98 & 1.77 \\
\hline polymer & -10.96 & -5.05 & -3.44 & 1.77 \\
\hline $\begin{array}{l}B_{\mathrm{w}}=5.91 \mathrm{eV} \\
E_{\mathrm{g}}=1.61 \mathrm{eV} \\
B_{\mathrm{w}}=5.21 \mathrm{eV}\end{array}$ & & & & \\
\hline
\end{tabular}

TABLE 2: Energies of Oligomer Orbitals Extrapolating toward Valence and Conduction Band Edges of PDFA in eV

\begin{tabular}{|c|c|c|c|c|}
\hline \# of carbons & $\begin{array}{c}\text { valence } \\
\text { band edge }\end{array}$ & HOMO & LUMO & $\begin{array}{l}\text { conduction } \\
\text { band edge }\end{array}$ \\
\hline 2 & & -8.50 & +0.14 & \\
\hline 4 & -10.08 & -7.89 & -1.65 & 1.74 \\
\hline 6 & -10.43 & -7.56 & -2.56 & 2.29 \\
\hline 8 & -10.55 & -7.35 & -3.08 & 2.52 \\
\hline 10 & -10.62 & -7.21 & -3.43 & 2.62 \\
\hline 12 & -10.66 & -7.11 & -3.66 & 2.68 \\
\hline 14 & -10.69 & -7.03 & -3.84 & 2.71 \\
\hline 16 & -10.71 & -6.98 & -3.98 & 2.73 \\
\hline 18 & -10.72 & -6.93 & -4.08 & 2.73 \\
\hline 20 & -10.74 & -6.89 & -4.16 & 2.74 \\
\hline 24 & -10.77 & -6.84 & -4.30 & 2.74 \\
\hline $\begin{array}{l}\text { polymer } \\
B_{\mathrm{w}}=4.25 \mathrm{eV} \\
E_{\mathrm{g}}=1.62 \mathrm{eV} \\
B_{\mathrm{w}}=7.69 \mathrm{eV}\end{array}$ & -10.82 & -6.57 & -4.95 & 2.74 \\
\hline
\end{tabular}

theory. The global minimum at the DFT level is a cis-transoid structure with a nearly $90^{\circ}$ twist between the terminal $\mathrm{CF}_{2}$ groups.

The order of the relative energies at the MP2 level does not agree with that at the DFT level. MP2 predicts the helical structure to be the global minimum, which corresponds to a change in relative energies of $3.90 \mathrm{kcal} / \mathrm{mol}$. At the MP2 level of theory, the energy between the planar all-trans structure and the global minimum is $5.44 \mathrm{kcal} / \mathrm{mol}$. We believe that the MP2 results are more reliable since DFT is known to have problems 


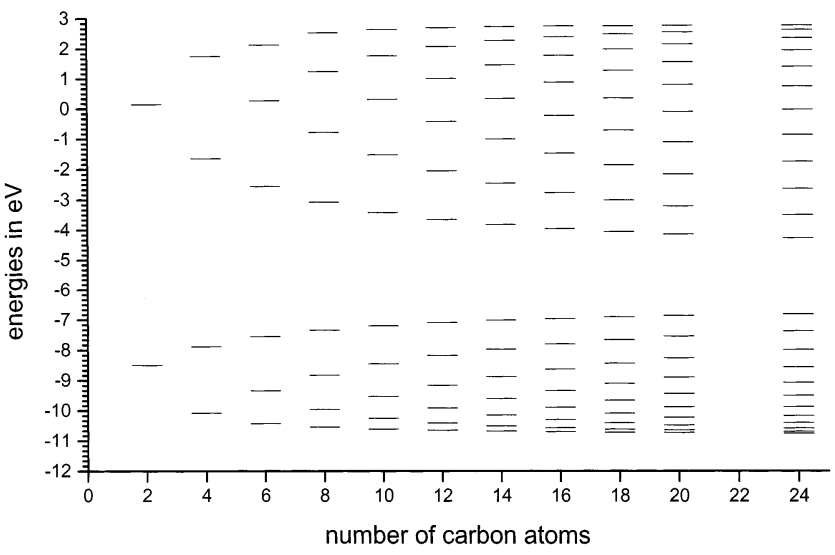

Figure 6. $\pi$-Energy levels of difluoroacetylene oligomers with 2-24 carbon atoms.

TABLE 3: Energies of Oligomer Orbitals Extrapolating toward Valence and Conduction Band Edges of PHFA in $\mathrm{eV}$

\begin{tabular}{lcccc}
\hline \# of carbons & $\begin{array}{c}\text { valence } \\
\text { band edge }\end{array}$ & HOMO & LUMO & $\begin{array}{c}\text { conduction } \\
\text { band edge }\end{array}$ \\
\hline 4 & -9.82 & -7.53 & -1.52 & 1.75 \\
8 & -10.50 & -6.86 & -2.69 & 2.35 \\
12 & -10.68 & -6.54 & -3.21 & 2.38 \\
16 & -10.76 & -6.36 & -3.51 & 2.37 \\
20 & -10.80 & -6.24 & -3.70 & 2.36 \\
24 & -10.83 & -6.16 & -3.83 & 2.34 \\
polymer & -10.96 & -5.74 & -4.51 & 2.22 \\
$B_{\mathrm{w}}=5.22 \mathrm{eV}$ & & & & \\
$E_{\mathrm{g}}=1.23 \mathrm{eV}$ & & & & \\
$B_{\mathrm{w}}=6.73 \mathrm{eV}$ & & & &
\end{tabular}

predicting dispersive forces. ${ }^{21}$ Thus, the most stable structure of DFOT is similar to that observed experimentally in crystals of the DCOT dianion. Since all structures have energies within $5.44 \mathrm{kcal} / \mathrm{mol}$, crystal-packing effects, which favor planar structures might influence the structure of PDFA in the solid state. A more planar geometry appears to be not impossible.

For DCOT (Figure 2) the relative energies of the nonplanar structures are similar to those of DFOT. Again DFT predicts the helical structure to lie too high in energy. The twisting of the trans-cisoid and cis-transoid structures is much stronger than for DFOT. The major difference between DFOT and DCOT is, however, that the planar trans structure is very unfavorable for DCOT. It lies $23.26 \mathrm{kcal} / \mathrm{mol}$ higher in energy than the global minimum at the MP2 level and has four imaginary frequencies at the DFT level. Thus repulsions between cyano groups appear to be much stronger than those between fluorine atoms.

For comparison, octatetraene was calculated at the same level of theory (Figure 3). Calculations at both DFT and MP2 levels of theory correctly predict the trans form to be the global minimum. Planar trans and cis-transoid forms are minima, having no imaginary frequencies. The trans-cisoid form is nonplanar. The planar form has three imaginary frequencies and lies $0.75 \mathrm{kcal} / \mathrm{mol}$ above the nonplanar minimum. The helical form has a $16.56 \mathrm{kcal} / \mathrm{mol}$ higher energy than the trans structure. Here the relative energy of the helical form is about $5 \mathrm{kcal} / \mathrm{mol}$ lower at the DFT level than at MP2.

Mixing difluoroacetylene with acetylene to form co-oligomers leads to a planar trans-conformer as the global minimum (Figure 4). The planar form lies $15.51 \mathrm{kcal} / \mathrm{mol}$ below the helical structure at the MP2 level of theory. Thus PFHA can be expected to form planar polymers analogous to PA.

\section{Discussion}

Our results for planar, all-trans PDFA confirm the earlier conclusions $^{7,8}$ regarding its low lying conduction band. This effect of fluorine substitution has also been noticed in experimental work on poly(difluoropyrrole), which has a lower redox potential than poly (dichloropyrrole). ${ }^{6}$ We do find, however, no reduction in band gap of PDFA compared to PA. The difference between this result and that of the earlier study ${ }^{7}$ might be due to the fact that the geometries had not been fully optimized. Optimization tends to increase the energy gap, since lowering the energy is achieved by lowering the energies of the occupied orbitals.

The increased electron affinity of PDFA was attributed to the electronegative character of fluorine. ${ }^{7}$ We do not believe that this is the correct explanation since ethylene and tetrafluoroethylene have quite similar HOMO and LUMO energies. The HOMO of tetrafluoroethylene lies $0.18 \mathrm{eV}$ lower and the LUMO lies $0.27 \mathrm{eV}$ higher in energy than that of ethylene. Two effects of fluorine substitution seem to cancel. First, fluorine has an electron withdrawing inductive effect. Electron withdrawal leads to stronger binding of the remaining electrons and therefore lowers the energies of the remaining electrons. Second, fluorine has a mesomeric effect since one of the lone pairs donates $\pi$-electron density to the $\pi^{*} \mathrm{C}=\mathrm{C}$ orbital. This increases the electron density in the $\pi$-system and therefore increases the energies of the $\pi$-electrons. This effect was also discussed by Yamabe et al. ${ }^{7}$ and Bakhshi et al. ${ }^{8}$ Since tetrafluoroethylene does not have a larger IP or a larger EA than ethylene, the increase in EA and IP of PDFA compared to PA occurs upon polymerization. This can be rationalized as follows. The inductive effect of fluorine leads to electron withdrawal from the $\sigma$-system and a stronger binding of the remaining electrons. This causes all occupied orbitals, including the HOMO, to contract. Upon polymerization, the overlap between the HOMOs of the tetrafluoroethylene repeat units is less compared to those of ethylene, and as a result the HOMO energy increases by only $1.93 \mathrm{eV}$. The corresponding value for PA is $3.27 \mathrm{eV}$. In contrast, the mesomeric effect of fluorine influences mainly the LUMO. This is reflected in the occupancies of the $\pi$-orbitals as revealed by NBO analysis. The $\pi$-lone pairs of fluorine are occupied by 1.94 rather than 2.00 e. The $\pi^{*} \mathrm{C}=\mathrm{C}$ orbital of tetrafluoroethylene has an occupancy of $0.24 \mathrm{e}$. Thus the LUMO of tetrafluoroethylene should be more diffuse than that of ethylene. Upon polymerization the overlap in the conduction band is larger in PDFA than in PA, and the increase in electron affinity is also larger, $5.09 \mathrm{eV}$ for PDFA compared to $3.31 \mathrm{eV}$ for PA. The increased overlap explains also the large bandwidth of the conduction band of PDFA. The described trends are clearly visible in Figures 5 and 6 . Thus, due to the mesomeric effect of fluorine, the EA of PDFA is large and the conduction band is exceptionally wide.

The stability of conducting polymers is influenced by the energies of the electrons in the valence and conduction bands. For instance, polypyrrole $(\mathrm{PPy})^{3}$ and poly(3,4-ethylenedioxythiophene) (PEDOT) ${ }^{22}$ have low IPs and are difficult to produce in their neutral forms. They are, however, very stable in their p-doped forms. Most conducting polymers have insufficiently large electron affinities to be stable in the n-doped form. Polythiophene (PTh), ${ }^{3}$ for instance, can be n-doped, but the $\mathrm{n}$-doped form is not stable enough to make PTh a good n-type conductor. An exception is poly(4-dicyanomethylene- $4 \mathrm{H}$-cyclopenta[2,1-b:3,4-b'] dithiophene) (PCDM) ${ }^{23}$ which can be repeatedly n-doped. Nonetheless, PCDM shows low conductivity in the n-doped form. Our hypothesis is that the lack of 
TABLE 4: Ionization Potentials and Electron Affinities as Obtained as Extrapolated Negative HOMO and LUMO Energies in eV

\begin{tabular}{lcc}
\hline & IP & EA \\
\hline PDFA & 6.57 & 4.95 \\
PA & 5.05 & 3.44 \\
PFHA & 5.74 & 4.51 \\
PPy & 4.77 & 1.61 \\
PTh & 5.50 & 3.20 \\
PEDOT & 4.38 & 2.34 \\
PCDM & 6.36 & 4.55
\end{tabular}

conductivity of the n-doped form of PCDM is caused by a lack of conjugation in the conduction band, which has a bandwidth of only $0.25 \mathrm{eV}$ according to our calculations at the same level of theory that was used here. ${ }^{24,25}$ Table 4 compares the negative valence and conduction band edge energies of PDFA and PFHA and of the above polymers. ${ }^{24-26}$ The values for PDFA are closest to those of PCDM. The EA of PFHA is almost identical to that of PCDM, the IP is closest to but above that of PTh. Compared to PA, which is difficult to handle because of its reactivity, PDFA and PFHA are a great improvement, since both have significantly higher IPs. Most important is that the low-lying conduction bands of PDFA and PFHA are very wide, 7.69 and $6.73 \mathrm{eV}$, respectively. Both systems might therefore be much better n-type conductors than PCDM.

A severe restriction might arise from the fact that PDFA has a tendency to be nonplanar. If PDFA remains helical in solution or in the solid state, it is probably useless as a conducting polymer. However, all structures of DFOT have energies within $5.44 \mathrm{kcal} / \mathrm{mol}$. Since crystal-packing favors planar structures, it seems possible that PDFA turns out to be planar or nearly planar in the solid state. Such a situation is observed, for instance, for thiophene oligomers, which are twisted in the gas phase $^{27}$ but perfectly planar in the crystal. ${ }^{28}$ The small energy difference between planar structure and global minimum is in contrast to that of DCOT for which the theoretical predictions of good conductivity were not borne out due to its nonplanarity. For DCOT, the planar form lies $23.26 \mathrm{eV}$ above the helical minimum. Moreover, all nonhelical forms are more strongly twisted than those of DFOT. Thus, PDCA has no chance of becoming planar under any circumstances. We believe that similar experimental problems will not necessarily arise for PDFA.

Alternating fluoroethylene and ethylene moieties lead to a planar structure for TFOT that lies $15.51 \mathrm{kcal} / \mathrm{mol}$ below the helical structure. This preference for planarity is almost as strong as that for OT. Therefore PFHA seems a good alternative, if PDFA turned out to be nonplanar. Since the band gap of PFHA is smaller than that of either PA or PDFA, it might even be the superior material. The amount of fluorine atoms in the chain can probably be used to fine-tune electronic properties between those of PDFA and PA. A final interesting aspect of this system is that one can expect strong hydrogen bonding in the condensed phase. This might lead to a strong, less disordered material.

Synthesizing the monomers for PDFA and PFHA might be challenging-some aspects were discussed by Yamabe et al. ${ }^{7}-$ since fluorinated alkenes have a tendency to polymerize explosively. However, it is encouraging to note that $1,1,4,4-$ tetrafluorobutadiene has been obtained recently ${ }^{29}$ and that hexafluorobutadiene can be produced at $30^{\circ} .{ }^{30}$

\section{Conclusions}

Electronic structures of PDFA and PFHA have been investigated. Owing to their wide low-lying conduction bands, both systems appear to be good candidates for $\mathrm{p}$ - and n-dopable materials with high on-chain electron mobility. Properties of mixed tetrafluoroethylene and ethylene polymers should be tunable between those of PDFA and PA depending on their fluorine content.

PDFA shows a tendency to adopt nonplanar and helical structures in the gas phase. Since energy differences are small, crystal-packing effects might overcome this problem. PFHA is fully planar and could be an alternative if PDFA retains the helical geometry in the solid state.

We believe that PDFA and PFHA are very promising materials and we have addressed some of the pitfalls theoreticians tend to overlook. It seems that the problems might be manageable and we hope that we stimulated interest with experimentalists to attempt making these compounds. Maybe a Teflon analogue of conducting polymers can be made.

Acknowledgment. The author thanks TÜBITAK (Grant: TBAG 1887) and Bilkent University for providing computational resources.

\section{References and Notes}

(1) Chiang, C. K.; Fincher, C. R.; Park, Y. W.; Heeger, A. J.; Shirakawa, H.; Louis, E. J.; Gao, S. C.; MacDiarmid, A. G. Phys. Rev. Lett. 1977, 39, 1098-1101.

(2) Naarmann, C. H.; Theophilou, N. Synth. Met. 1987, 22, 1.

(3) Handbook of Conducting Polymers, second ed.; Skotheim, T. A., Elsenbaumer, R. L., Reynolds, J. R., Eds.; Marcel Dekker: New York, 1997.

(4) Heidenhain, S. B.; Sakamoto, Y.; Suzuki, T.; Miura, A.; Fujikawa, H.; Mori, T.; Tokito, S.; Taga, Y. J. Am. Chem. Soc. 2000, 122, 1024010241.

(5) Sakamoto, Y.; Komatsu, S.; Suzuki, T. J. Am. Chem. Soc. 2001, $123,4643-4644$.

(6) Audebert, P.; Miomandre, F.; Di Magno, S. G.; Smirnov, V. V.; Hapiot, P. Chem. Mater. 2000, 12, 2025-2030.

(7) Yamabe, T.; Tanaka, K.; Terama-E, H.; Fukui, K.; Shirakawa, H.; Ikeda, S. Synth. Met. 1980, 1, 321-327.

43

(8) Bakhshi, A. K.; Ladik, J.; Liegener, C.-M. Synth. Met. 1987, 20,

(9) Abdelaty, S. M.; Fukutome, H. Prog. Theor. Phys. 1986, 75, 12831294.

(10) Yu, H.; Srdanov, G.; Hasharoni, H.; Wudl, F. Tetrahedron 1997, $45,15593-15602$.

(11) Becke, A. D. Phys. Rev. A 1988, 38, 3098-3100.

(12) Perdew, J. P. Phys. Rev. B 1986, 33, 8822-8824.

(13) Stevens, W.; Basch, H.; Krauss, J. J. Chem. Phys. 1984, 81, 6026.

(14) Salzner, U.; Lagowski, J. B.; Pickup, P. G.; Poirier, R. A. J. Phys. Chem. 1998, 102, 2572-2578.

(15) Brunck, T. K.; Weinhold, F. J. Am. Chem. Soc. 1978, 100, 1700.

(16) Foster, J. P.; Weinhold, F. J. Am. Chem. Soc. 1980, 102, 7211.

(17) Reed, E. A.; Weinstock, R. B.; Weinhold, F. J. Chem. Phys. 1985, 83,735 .

(18) Reed, A. E.; Weinhold, F. J. Chem. Phys. 1985, 83, 1736.

(19) Reed, E. A.; Curtiss, L. A.; Weinhold, F. Chem. Rev. 1988, 88, 899.

(20) Frisch, M. J.; Trucks, G. W.; Schlegel, H. B.; Scuseria, G. E.; Robb, M. A.; Cheeseman, J. R.; Zakrzewski, V. G.; Montgomery, J. A., Jr.; Stratmann, R. E.; Burant, J. C.; Dapprich, S.; Millam, J. M.; Daniels, A. D.; Kudin, K. N.; Strain, M. C.; Farkas, O.; Tomasi, J.; Barone, V.; Cossi, M.; Cammi, R.; Mennucci, B.; Pomelli, C.; Adamo, C.; Clifford, S.; Ochterski, J.; Petersson, G. A.; Ayala, P. Y.; Cui, Q.; Morokuma, K.; Malick, D. K.; Rabuck, A. D.; Raghavachari, K.; Foresman, J. B.; Cioslowski, J.; Ortiz, J. V.; Stefanov, B. B.; Liu, G.; Liashenko, A.; Piskorz, P.; Komaromi, I.; Gomperts, R.; Martin, R. L.; Fox, D. J.; Keith, T.; Al-Laham, M. A.; Peng, C. Y.; Nanayakkara, A.; Gonzalez, C.; Challacombe, M.; Gill, P. M. W.; Johnson, B. G.; Chen, W.; Wong, M. W.; Andres, J. L.; Head-Gordon, M.; Replogle, E. S.; Pople, J. A. Gaussian 98, revision A.11.2; Gaussian, Inc.: Pittsburgh, PA, 1998.

(21) Koch, W.; Holthausen, M. C. A Chemist's Guide to Density Functional Theory; Wiley: Weinheim, 2000.

(22) Groenendaal, L.; Lonas, F.; Freitag, D.; Pielartzik, H.; Reynolds, J. R. Adv. Mater. 2000, 12, 481-494. 1268. 
(24) Salzner, U. J. Mol. Model. 2000, 6, 195-204.

(25) Salzner, U.; Köse, M. E. J. Phys. Chem. B 2002, 106, 9221-9226.

(26) Salzner, U.; Lagowski, J. B.; Poirier, R. A.; Pickup, P. G. Synth. Met. 1998, 96, 177-189.

(27) Duarte, H. A.; dos Santos, D. A.; Rocha, W. R.; de Almeida, W. B. J. Chem. Phys. 2000, 113, 4206-4215.
(28) Horowitz, G.; Bachet, B.; Yassar, A.; Lang, P.; Demanze, F.; Fave, J.-L.; Garnier, F. Chem. Mater. 1995, 7, 1337-1341.

(29) Bach, A.; Lentz, D.; Luger, P.; Messerschmidt, M.; Olesch, C.; Patzschke, M. Angew. Chem., Int. Ed. Engl. 2002, 41, 296-299.

(30) Vandersypen, L. M. K.; Steffen, M.; Breyta, G.; Yannoni, C. S.; Sherwood, M. H.; Chuang, I. L. Nature 2001, 414, 883-887. 